\title{
A NEW, MODERN, COST-SAVING MICRO/MACRO METHOD FOR THE DETERMINATION OF SERUM FRUCTOSAMINE
}

\author{
Klára OPPEL ${ }^{1 *}$, Margit KULCSÁR ${ }^{2}$, L. BÁRDos ${ }^{1}$, A. FERENCZ ${ }^{3}$, Hajnalka LAKNER ${ }^{1}$, \\ Judit SimON $^{3}$, Kriszta TEMESVÁRY ${ }^{1}$ and Krisztina KARCHESZ ${ }^{1}$ \\ ${ }^{1}$ Department of Animal Physiology and Health, Faculty of Agriculture and Environ- \\ mental Science, Szent István University, H-2103 Gödöllő, Páter Károly u. 1, Hungary; \\ ${ }^{2}$ ELISA Laboratory of the Department of Obstetrics and Reproduction, \\ Faculty of Veterinary Science, Szent István University, Budapest, Hungary; \\ ${ }^{3}$ Clinical and Experimental Medical Laboratory Institute, 'Imre Haynal' Faculty of \\ Human Health Sciences, Semmelweis University, Budapest, Hungary
}

(Received September 3, 1999; accepted May 3, 2000)

Serum/plasma fructosamine $(\mathrm{SeFa})$ concentration is a reliable indicator used in human diabetic control. Tests for monitoring the carbohydrate/energy metabolism of (farm) animals are less commonly performed in veterinary laboratories, since most of the reliable determinations, both automated and manual, are relatively expensive. The aim of this study was to develop a precise, money- (and time-) saving automated micro method for measuring SeFa. ELISA microplates (20 $\mu \mathrm{L}$ samples and $200 \mu \mathrm{L}$ reagents) and an automatic microplate autoreader were used. The classical nitroblue tetrazolium (NBT) stain reagent solution of Johnson et al. (1982) was modified using a SIGMA reagent to render it stable for up to one year. SeFa concentrations measured by the new method in 30 human blood plasma samples were compared with values obtained by the standard (generally used) LaRoche kit procedure. Fifteen cow, 13 dog and 18 chicken plasma samples were assayed by the new automated 'micro' method as well as by the manual test tube 'macro' method commonly used earlier. The modified reagent was applied for both methods. The coefficient of correlation (r) between the results obtained by the two methods was consistently between 0.94 and 0.98 $(\mathrm{p}<0.001)$.

Key words: Serum fructosamine, glycated proteins, glycated haemoglobin, new automated micro method, cost-saving modified reagent

Blood parameters used in clinical laboratory investigations and for nutritional monitoring and profile tests include important plasma proteins, lipids and other constituents. Blood glucose is one of the most sensitive parameters for evaluating the carbohydrate metabolism of domestic animals and humans. However, it is well known (Andresen, 1977) that this parameter does not reflect with full precision the actual status and alterations of carbohydrate metabolism, e.g.

"E-mail: oppel@fau.gau.hu; Fax: +36 (28) 410804 
OPPEL et al.

the effect of nutritional problems, diabetes, or the hyperglycaemic effect occurring after adrenalinaemia caused by sympathicotonia. Even the process of withdrawing blood samples may elevate the existing blood glucose level via the above mechanism.

This is why glycated blood parameters [the $\mathrm{Hb}_{\mathrm{Alc}}$ subfraction of glycated haemoglobin $(\mathrm{GHb})$ and serum fructosamine $(\mathrm{SeFa})]$ were introduced (Rahbar, 1968) and have been used routinely since the mid-eighties as reliable indicators in human diabetic control (Miedema and Casparie, 1984; Austin et al., 1987). Their physiological values measured in healthy adult individuals $\left(4-6 \%\right.$ for $\mathrm{Hb}_{\mathrm{Alc}}$ and $2.0-2.8 \mathrm{mmol} / \mathrm{L}$ for $\mathrm{SeFa}$ ) might rise significantly in diabetic patients, reflecting the actual blood glucose level in the previous 4 and 2 weeks, respectively.

Few data can be found in the literature on these parameters in connection with diabetes in animals (Mahaffey et al., 1984; Staudacher, 1990), and reports are especially scarce as regards farm animals such as cattle (Ropstad, 1988; Bárdos and Oppel, 1989; Oppel et al., 1999-2000), horses and poultry (Oppel et al., 2000). Moreover, animal studies concern mostly the determination of SeFa. Studies available on the glycosylation (glycation) of blood proteins in domestic animals, indicative of chronic energy deficiency (critically low blood glucose in bovine ketosis) and energy overfeeding in horses, and the physiological basic values of different animal species have been summarised in a review article (Oppel et al., 2000).

The formation of glycoproteins is known to be a genetically regulated process. In these conjugated proteins a carbohydrate group is linked to a polypeptide chain with the help of several enzymes. In contrast, glycation (or glycosylation) of proteins is an entirely different process. The end-product of this latter process is fructosamine. A post-translational, non-enzymatic glycosylation takes place in the blood plasma, so that glucose binds to the plasma proteins in two steps. By the uptake of one water molecule, the two components first form aldimine (Schiff-base) through a reversible process. Subsequently, the stable ketoamine, fructosamine is formed after the so-called Amadori rearrangement (Baynes et al., 1984; Roche, 1989). Albumin represents $80 \%$ of the glycated serum proteins (Schleicher et al., 1988). In humans, glycation involves the $\varepsilon$-amino groups of the N-terminal lysine residues of albumin and other proteins in the blood plasma and other tissues (Baynes et al., 1984; Roche, 1989). Similarly, glycated haemoglobin is formed by the binding of glucose to the N-terminal valine of the haemoglobin $\beta$-chains.

The practical importance of measuring glycated proteins is that their percentage in the blood depends on the concentration of blood glucose. The glucoseprotein complexes (glycated proteins) are more stable than the continuously changing blood glucose levels. Fructosamine concentration depends on the halflife of the protein (Woo et al., 1987), while glycated haemoglobin persists even longer, throughout the life span of red blood cells, though its life span varies by 
species. That is why fructosamine and glycated haemoglobin are good indicators of blood glucose levels (depending on the species) of the previous 5-14 days and 2-4 weeks, respectively. Monitoring of these parameters is already used routinely in the clinical laboratory control of human diabetes. However, veterinary laboratories should also increase the number of tests aimed at the laboratory monitoring of small animal diabetes, and should introduce methods for evaluating the carbohydrate and energy metabolism of other mammals and poultry.

The relatively low number of animal analyses described in the literature may be due to methodological problems. Even the determination of serum/plasma fructosamine, which does not need a species-specific determination method as does haemoglobin, is less widely applied, because most of the reliable methods (automated or manual) are relatively expensive.

The aim of this study was to develop a precise, money- (and time-) saving automated method for the measurement of SeFa. The classical method of Johnson et al. (1982), the nitroblue tetrazolium (NBT) method, was modified. This decision was in good agreement with the efforts of others to use an automated and easily performed colorimetric method based on the use of nitroblue tetrazolium reagent for the determination of $\mathrm{SeFa}$.

The reaction is based on the principle that in alkaline media fructosamine reduces NBT to form formazan. Precise SeFa test kits manufactured by leading laboratory diagnostic firms are available on the market. Their disadvantages are the requirement for special automated equipment and the high reagent costs due to the need for specific and complex stabilisation processes (uricase complementation, use of detergent) during production of the reagent solution by the manufacturer.

\section{Materials and methods}

In this study, development of the reagent required only a few minor chemical modifications of the original method of Johnson et al. (1982), as described below. Another difference is the miniaturisation of the method, so that it can be applied on ELISA plates without special laboratory equipment or machines.

\section{Reagent}

Fifty mg NBT stain (SIGMA) (nitroblue tetrazolium grade III, crystalline, Mw 817.6 g, lot no. 77H5083) was dissolved in $244.6 \mathrm{~mL}$ carbonate buffer at $\mathrm{pH}=10.11$. To producing of the buffer were mixed $13.375 \mathrm{~g} / 250 \mathrm{~mL}(0.5 \mathrm{Mol})$ $\mathrm{Na}_{2} \mathrm{CO}_{3}$ and $2.1 \mathrm{~g} / 50 \mathrm{ml}(0.5 \mathrm{Mol}) \mathrm{NaHCO}_{3}$. The $\mathrm{pH}(10.11)$ may be corrected with $1 \mathrm{n} \mathrm{NaOH}$ solution, if necessary. When stored at $+4{ }^{\circ} \mathrm{C}$, the reagent produced in this way retained the stability of its yellow colour and provided highly reproducible measurement results for 12 months. 
OPPEL et al.

\section{Methodology}

Experiment 1. ELISA microplates (CEB, France) with 96 U-formed wells were used for the new 'micro' method. $20 \mu \mathrm{L}$ plasma and standard samples were pipetted into the wells separately in three parallels, using Finnpipettes. Subsequently $200 \mu \mathrm{L}$ aliquots of reagents were measured into the wells with an eightchannel pipette. The plates were incubated in a shaking machine (Amersham) at $37^{\circ} \mathrm{C}$ for $5 \mathrm{~min}$, then read in an automatic microplate autoreader (EL 311, Bio-Tek Instruments) at $550 \mathrm{~nm}$, and evaluated automatically (Okidata Microline 184 Turbo 9 Pin Printer), to determine the initial absorbance $\left(\mathrm{A}_{1}\right)$. The plates were again incubated for $5 \mathrm{~min}$, and after determination in the same way the $A_{2}$ values were measured. The concentrations of the samples were determined from differences of the two values of absorbance, with the help of the known standard values.

Mostly fresh or at most 1 -week-old refrigerated $\left(-20^{\circ} \mathrm{C}\right)$ blood plasma samples were used for the measurements. The time of storage was the same within each series. Well-controlled human plasma samples (i.e. freshly determined with the LaRoche kit by the closest 'reference laboratory') were used as standard (one sample per analysis in four wells). Each of these standard samples proved to be accurate within 1 week (divided and stored at $-20{ }^{\circ} \mathrm{C}$ in small quantities till each day of analysis). This allowed us to save the high costs of manufactured standards. Reference (control or split) samples, i.e. pooled plasma samples previously assayed by our laboratory, were also used in four wells on every plate to increase the reliability of the assay.

As the four wells at the edges of the plates were filled with distilled water, the remaining 84 wells made it possible to determine SeFa in 28 unknown plasma samples, working with three sample parallels.

\section{Results and discussion}

SeFa concentrations measured in 30 human samples by the standard LaRoche kit method $(2.0-5.5 \mathrm{mmol} / \mathrm{L})$ were compared with the values obtained by this new micro method. Figure 1 shows the close correlation of plasma fructosamine concentrations $(r=0.98 ; y=0.94+0.25 \mathrm{x} ; \mathrm{p}<0.001)$ obtained by the two methods.

The standard deviation of the results and the coefficient of variation (CV\%, the error of the method) were calculated from three parallels of each plasma sample on the plate. The CV\% was generally less than $2.5 \%$. Thus, the $\mathrm{SeFa}$ concentrations obtained by the micro method differed by $0.30-2.63 \%$ from those measured by the LaRoche kit procedure. As the use of day-to-day plate controls did not result in higher errors, one standard proved to be sufficient if the reference sample also showed the accurate level. In other cases the use of two or three standards is recommended. 


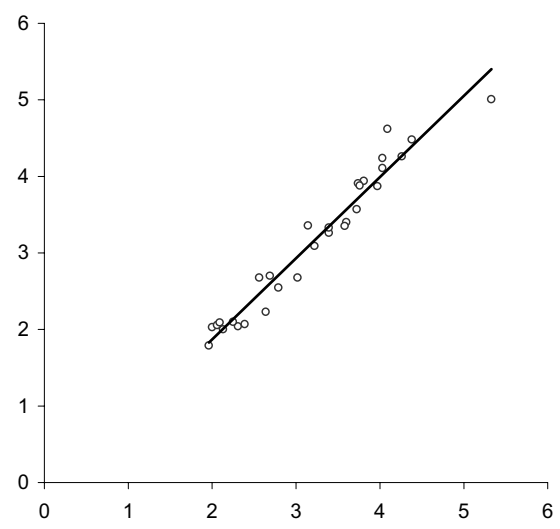

Fig. 1. Correlation between human plasma fructosamine concentrations $(\mathrm{mmol} / \mathrm{L} ; \mathrm{n}=30)$ measured by the standard LaRoche Kit method (x axis) and those determined by the new micro method (y axis): $y=0.2518+0.9437 x ; r=0.9816(p<0.001)$

\section{Experiment 2}

Earlier the kit manufactured by Reanal (Hungary; Szilágyi et al., 1985) was used for the determination. Using the same quantities of materials of that and of our modified reagent, we developed the following modification termed 'macro method'. First $2000 \mu \mathrm{L}$ NBT reagent and then $200 \mu \mathrm{L}$ serum or plasma sample was pipetted into glass test tubes. The two absorbance values of the samples and standards $\left(A_{1}\right.$ and $\left.A_{2}\right)$ were measured after the same incubation times and manual photometer (Zeiss, Germany) procedure as described above for the micro method. The procedure and the results obtained for human samples proved to be the same with the kit and with our modified, home-made reagent.

In Experiment 2, 15 cow, 13 dog and 18 chicken samples were assayed using the modified reagent described above, both by the new 'micro' and the 'macro' method. The SeFa concentrations were 1.90-2.55, 1.35-1.75 and 2.29$3.07 \mathrm{mmol} / \mathrm{L}$ in these cow, dog and chicken samples, respectively. The coefficients of correlation ( $\mathrm{r}$ ) between results obtained by the two methods were consistently between 0.94 and $0.98(\mathrm{p}<0.001)$. It has to be mentioned that in this case our aim was to perform a methodological comparison, rather than to determine the physiological SeFa concentrations of the above-mentioned animal species. These samples were obtained from specific experiments, with the actual characteristic feeding, health and husbandry circumstances.

The reaction has only one 'critical point' which, however, has to be taken very seriously. It is the incubation time of the mixed reagent and plasma samples, which must be the same for each sample of the investigated series. If the laboratory has facilities for the almost simultaneous automatic evaluation of all the samples, first the samples and then the reagents should be applied onto the 
ELISA plate. If the laboratory has only a semi-automatic (manual) microplate reader, first the reagents and then the samples should be pipetted into the wells, one after the other, as in the case of the test tube macro method, and evaluation should also follow in that sequence.

The newly developed micro method using $20 \mu \mathrm{L}$ of blood plasma proved to be rapid, accurate and highly reproducible. The reagents used for determining the $\mathrm{SeFa}$ concentration of one plasma sample in three parallels cost only $2 \%$ of the costs of assaying one plasma sample by the manufacturer's test. The new method also facilitates better utilisation of equipment in modern ELISA laboratories, even if a large number of samples have to be tested.

Small veterinary laboratories equipped with a photometer can also use the manual macro (test tube) method for testing a small number of samples daily, using a fresh plasma obtained from the nearest human reference laboratory as standard. In this case the terms 'reference laboratory' and 'well-controlled samples' shall be interpreted as the nearest leading laboratory of a given region, which provides accurate results on fresh measured samples. Co-operation between laboratories diminishes the need of purchasing manufactured standard samples in each case.

The method can safely be recommended for veterinary laboratories, as it can reliably detect chronic energy deficiency in cattle and overfeeding in horses (Oppel et al., 2000).

Determination of fructosamine in human serum samples has successfully been used for diabetes mellitus monitoring since the mid-eighties. However, according to Eder (1997) the fructosamine test used until now had $97 \%$ sensitivity and $77 \%$ specificity for diabetes. Another yet unsolved problem is the extremely poor standardisation of human fructosamine tests. By the new approach to standard samples, our aim was to save the high costs of manufactured standards. A system of links among the best, so-called 'reference' laboratories and the close collaboration of clinical laboratories of a given region could enable the use of the method not only in veterinary laboratories but (with the parallel application of manufactured standards) perhaps also in human laboratory determinations.

With the manual 'macro' method the quantity of samples and reagents is tenfold as compared to the 'micro' method. However, this method is also economical, since $50 \mathrm{mg}$ of NBT dye (SIGMA, 9.98 EUR) dissolved in $244.6 \mathrm{~mL}$ of inexpensive buffer enables the accurate determination of SeFa concentration in about 120 samples, and the stability of the reagent allows its use for up to one year.

The new method enables most veterinary laboratories to perform diabetes control tests in dogs and cats and to monitor carbohydrate and energy metabolism in other mammals and poultry. Its two varieties (ELISA micro method and test tube macro method) make the procedure suitable for use by well-equipped clinical laboratories and smaller laboratories alike. 


\section{Acknowledgement}

The authors thank SIGMA-ALDRICH Ltd., Budapest for providing the reagent used for the development of the new method.

\section{References}

Andresen, E. (1977): Dukes' Physiology of Domestic Animals. In: Swenson, M. J. (ed.) Cornell University Press, Ithaca and London. p. 48.

Austin, G. E., Mullins, R. H. and Morin, L. G. (1987): Non-enzymic glycation of individual plasma proteins in normoglycemic and hyperglycemic patients. Clin. Chem. 33, 2220-2224.

Baynes, J. W., Thorpe, S. R. and Murtiashaw, M. H. (1984): Nonenzymatic glucosylation of lysine residues in albumin. Meth. Enzymol. 106, 88-98.

Bárdos, L. and Oppel, K. (1989): Determination of glycohaemoglobin levels in ketonuric and nonketonuric cows (Short communication). Acta Vet. Hung. 37, 299-301.

Eder, A. F. (1997): Laboratory tests for glycaemic control in diabetes. Clinical Chemistry Check Sample CC. Chicago IL Ascp. 97-98.

Johnson, R. N., Metcalf, P. A. and Baker, J. R. (1982): Fructosamine: a new approach to the estimation of serum glycosylprotein. An index of diabetic control. Clin. Chim. Acta 127, 87-95.

Mahaffey, E. A., Buonanno, A. M. and Cornelius, L. M. (1984): Glycosylated albumin and serum protein in diabetic dogs. Am. J. Vet. Res. 45, 2126-2128.

Miedema, K. and Casparie, T. (1984): Glycosylated hemoglobins: biochemical evaluation and clinical utility. Ann. Clin. Biochem. 21, 2-15.

Oppel, K., Bárdos, L., Lakner, H., Koch, A. and Varga, P. (1999-2000): Glycated haemoglobin $(\mathrm{GHb})$, blood sugar, adult and fetal haemoglobin (HbA, HbF) levels in cattle and calves. Bull. Univ. Agric. Sci. Gödöllő (in press).

Oppel, K., Bárdos, L., Lakner, H., Temesváry, K., Bölcsházy, G., Kulcsár, M., Ferencz, A. and Simon, J. (2000): Glucosylated (glycated) proteins and the importance of their investigation in domestic animals: Literature review (in Hungarian, with English abstract). Magyar Állatorvosok Lapja 122, 106-111.

Rahbar, S. (1968): An abnormal hemoglobin in red cells of diabetics. Clin. Chim. Acta 22, 296-299.

Roche Diagnostics (1989): Fructosamine test plus. Instruction Manual. Roche, Basel, Switzerland.

Ropstad, E. (1988): Constituents of blood and milk in relation to fertility, nutrition and metabolic status in dairy cows. PhD Dissertation and Thesis. Norwegian College of Veterinary Medicine, Oslo.

Schleicher, E. D., Mayer, R., Wagner, E. M. and Gerbitz, K. D. (1988): Is serum fructosamine assay specific for determination of glycated serum protein? Clin. Chem. 34, 320-323.

Staudacher, G. (1990): Fructosamine, a new valuable criterion for the treatment of diabetes mellitus in animals and its photometric determination (in German, with English abstract). Tierärztl. Praxis 18, 441-446.

Szilágyi, L., Lendvai, B., Németh, Á. and Töke, I. (1985): Determination of glycosylated protein with the application of 2-amino-2-methyl-1-propanol-hydrochloric acid buffer (Reanaltest) (in Hungarian, with English abstract). Laboratóriumi Diagnosztika XII:2. 34.

Woo, J., Weinstock, R. S., Ozark, C. and Sunderji, S. J. (1987): Glycated albumin by affinity chromatography and radioimmunoassay in the management of diabetes mellitus. J. Clin. Lab. Anal. 1, 163-169. 